\title{
Statistical characterization of dislocation ensembles
}

Anter El-Azab, Jie Deng, Meijie Tang

May 23, 2006

Statistical Characterization of Dislocation Ensembles 
This document was prepared as an account of work sponsored by an agency of the United States government. Neither the United States government nor Lawrence Livermore National Security, LLC, nor any of their employees makes any warranty, expressed or implied, or assumes any legal liability or responsibility for the accuracy, completeness, or usefulness of any information, apparatus, product, or process disclosed, or represents that its use would not infringe privately owned rights. Reference herein to any specific commercial product, process, or service by trade name, trademark, manufacturer, or otherwise does not necessarily constitute or imply its endorsement, recommendation, or favoring by the United States government or Lawrence Livermore National Security, LLC. The views and opinions of authors expressed herein do not necessarily state or reflect those of the United States government or Lawrence Livermore National Security, LLC, and shall not be used for advertising or product endorsement purposes. 


\title{
Statistical characterization of dislocation ensembles
}

\author{
ANTER EL-AZAB ${ }^{1} \dagger$, JIE DENG†and MEIJIE TANG $\ddagger$ \\ $†$ Mechanical Engineering Department, FAMU-FSU college of \\ Engineering, Florida State University, Tallahassee FL 32310, US \\ łLawrence Livermore National Laboratory, 7000 East Avenue, \\ Livermore, CA 94550, US
}

(Received May 2006)

\begin{abstract}
We outline a method to study the spatial and orientation statistics of dynamical dislocation systems by modeling the dislocations as a stochastic fiber process. Statistical measures have been introduced for the density, velocity, and flux of dislocations, and the connection between these measures and the dislocation state and plastic distortion rate in the crystal is explained. A dislocation dynamics simulation model has been used to extract numerical data to study the evolution of these statistical measures numerically in a body-centered cubic crystal under deformation. The orientation distribution of the dislocation density, velocity and dislocation flux, as well as the dislocation correlations have been computed. The importance of the statistical measures introduced here in building continuum models of dislocation systems is highlighted.
\end{abstract}

\section{Introduction}

The plastic distortion of crystals proceeds by crystallographic slip, which is carried by glide of crystal dislocations under the influence of applied load. The process of crystallographic slip enters the crystal plasticity theory through the continuum kinematics and constitutive laws; a set of shear strain rates defined for the individual slip systems are superposed to give the rate of plastic distortion of the crystal, and these strain rates are determined in terms of the local resolved shear stress on the

\footnotetext{
${ }^{1}$ Corresponding author. Email: anter@eng.fsu.edu
} 
respective crystallographic slip planes $[1,2]$. At the mesoscale, however, the plastic distortion of crystals is highly heterogeneous, an important feature that cannot be captured by the crystal plasticity theory. Attempts to formulate a crystal plasticity theory based upon direct modeling of dislocations led to the development of the classical theory of dislocation fields $[3,4]$. In this theory, dislocations are modeled by a density tensor $\boldsymbol{\alpha}$, which, in the case of infinitesimal distortion, is defined by

$$
\boldsymbol{\alpha}=\operatorname{curl} \boldsymbol{\beta}^{p}
$$

where $\boldsymbol{\beta}^{p}$ is the plastic distortion. The sum of $\boldsymbol{\beta}^{p}$ and its elastic counterpart $\boldsymbol{\beta}^{e}$ gives the continuum displacement gradient. By definition, the dislocation density tensor is divergence free and its evolution is described by the rate form of equation (1) [5]. In terms of crystal dislocations, the density tensor is represented by

$$
\boldsymbol{\alpha}=\sum_{n=1}^{N} \int_{4 \pi} \varrho^{(n)}(\boldsymbol{m})\left(\boldsymbol{m} \otimes \mathbf{b}^{(n)}\right) d \Omega(\boldsymbol{m})
$$

in which $N$ is the number of slip systems in the crystal, $\mathbf{b}^{(n)}$ and $\varrho^{(n)}(\boldsymbol{m})$ are the Burgers vector and scalar density of dislocations with unit tangent $\boldsymbol{m}$ on the $n^{\text {th }}$ slip system, respectively, and $d \Omega(\boldsymbol{m})$ is a differential solid angle element in the direction $\boldsymbol{m}$. The classical theory of dislocation fields needs a closure constitutive law connecting the rate of plastic distortion $\dot{\boldsymbol{\beta}}^{p}$ with the local resolved shear stress. The task of developing this constitutive law, however, proved to be difficult because the representation of the evolution of the dislocation field by the dislocation density tensor alone is inadequate.

The invention of the dislocation dynamics simulation method about two decades ago to model the collective behavior of dislocations and mesoscale plasticity represents a turning point in the field - relevant $3 \mathrm{D}$ models can be found in $[6,7,8]$. The method represents the dislocation lines in a discrete fashion by dividing these lines into a finite number of connected segments the end or middle points of which are assigned velocity values that depend on the local stress at these points. The velocity is then used to update the position of the nodes or segments, thus updating the entire dislocation 
configuration. The rate of plastic distortion is computed using Orowan's formula,

$$
\dot{\boldsymbol{\beta}}^{p}(\mathbf{x}, t)=\sum_{n=1}^{N} \int_{l^{(n)}(t)} \delta\left(\mathbf{x}-\mathbf{x}^{\prime}\right)\left(d \mathbf{x}^{\prime} \times \mathbf{v}\left(\mathbf{x}^{\prime}, t\right)\right) \otimes \mathbf{b}^{(n)}
$$

in which $l^{(n)}(t)$ is union of all dislocation lines on $n^{t h}$ slip system, $\delta\left(\mathbf{x}-\mathbf{x}^{\prime}\right)$ is the $3 \mathrm{D}$ dirac-delta distribution, and $\mathbf{v}$ is the dislocation velocity. Equation (3) thus shows that $\dot{\boldsymbol{\beta}}^{p}$ is non-trivial only along the line indicating its statistical character, which is a consequence of the statistical line distribution. Having its long range part determined by the system of dislocation lines, the stress field is also a statistical field. Ensemble averaging or running volume averaging process can be used to extract continuous approximations of these fields. Thus the method of dislocation dynamics simulation is a statistical method.

The use of statistical mechanics concepts to describe the collective behavior of dislocations was first suggested by Kröner who argued that the dislocation state of a deformed body represents its internal mechanical state and that the incomplete information about the dislocation state permits only probabilistic predictions $[9,10]$. Lately, a statistical framework along the lines of the classical kinetic theory has been developed to model the collective behavior of 3D dislocation systems [11]; see also $[12,13]$. In this framework, the dynamical dislocation system comprises a set of families of planar curves, one family per slip system, each of which is represented by a dislocation phase density $\psi^{(\alpha)}(\mathbf{x}, \mathbf{v}, \theta, t)$ in a $7 \mathrm{D}$ phase space that consists of the real crystal space, the velocity space and the orientation space represented by an angle $\theta$ describing the dislocation orientation in its slip plane. This kinetic approach thus differentiates between the dislocation segments based on their line orientation, which is required to avoid the difficulties that arise in connecting the dislocation density evolution measure, such as the rate of plastic distortion (or dislocation flux tensor), with the local stress state in the classical theory of dislocation fields. In order to develop a complete theory based on this representation of the dislocation system, four types of dislocation statistics must be modeled: spatial statistics, velocity statistics, orientation statistics, and temporal statistics. These types of statistics have been pointed out in Ref. [14], where the connection between the above phase density 
representation of dislocations and the concept of a stochastic fiber process was also highlighted.

This paper builds upon the statistical approach presented in Refs. $[11,14]$. A mathematical description of the spatial and orientation statistics of evolving dislocation systems is introduced in section 2 based on the concept of stochastic fiber process [15]. Statistical measures are given for the dislocation density, dislocation correlations, velocity and the dislocation flux. The connection between statistical measures of the velocity and flux) and the strain rate in deforming crystal is explained. Section 3 contains numerical simulations of the above statistical measures that were conducted using the ParaDis dislocation dynamics code [16]. A discussion of the important findings of this study and the relevance of the study to the development of a continuum theory of dislocations is given in section 4 .

\section{Theoretical foundation}

\subsection{Dislocations as spatial curves}

During plastic distortion under a general loading, dislocations of all Burgers vectors increase in density. Because of the strong long-range interactions, cross slip and the reactions at short range, the evolution of the density in the crystal is heterogeneous. The dislocation dynamics simulation methods, part of the numerical tools used in this work, can reveal the nature of the heterogeneity of the dislocation density evolution. Figure 1 shows a typical dislocation density distribution in a body-centered cubic crystal loaded in tension to a strain of 0.018 at elevated temperature. The crystal has a shear modulus of $130 \mathrm{GPa}$ and a poisson's ratio of 0.31 ; see the ParaDis model details in section 3.1 .

Add figure 1 here.

If dislocation motion is restricted to glide in the slip planes, then the dislocation system consists of a set of planar curves. This kind of motion is termed conservative. On the other hand, if the dislocation motion has both glide and non-glide 


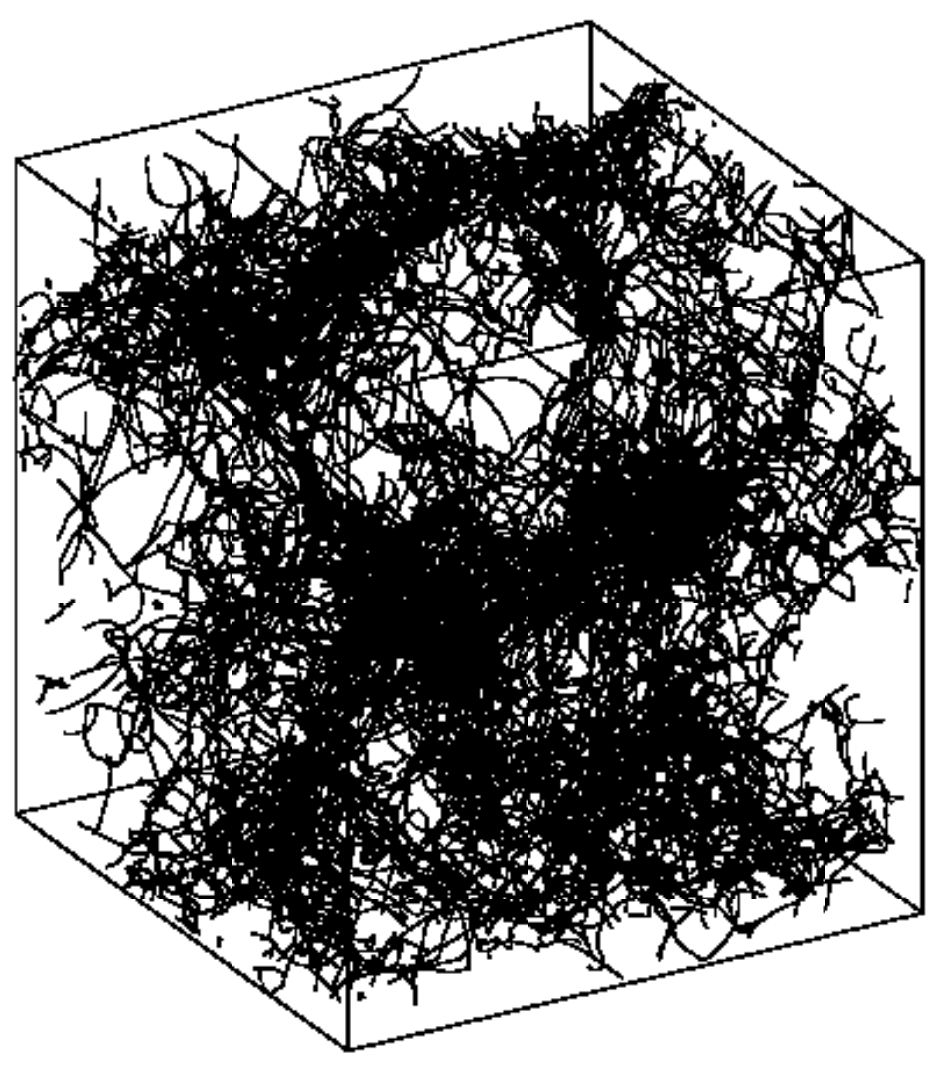

Figure 1: Snapshot of the dislocation distribution in Nb crystal loaded in the [100] direction at constant strain rate of $1 \mathrm{~s}^{-1}$ (strain $\left.=0.018\right)$. 
components, that is, dislocations are permitted to move off their glide planes, then the dislocation system consists of a set of spatial (3D) curves. The latter motion is termed nonconservative. Irrespective of the type of motion, when the glide planes undergo nonuniform finite lattice rotation, the dislocation lines are no longer planar. For a given Burgers vector, the dislocation system includes dislocation lines on more than one cross slip plane, and thus consists of spatial curves.

\subsection{Stochastic fiber process}

The definition of stochastic fiber process introduced here is adopted from Ref. [15]. The set theory terminology required to understand this section are compiled in the appendix.

A spatial fiber is a smooth simple curve of finite length, which is a subset of $\mathbb{R}^{3}$. It is the image of a curve $l$ spanned by a parameterized position vector $\mathbf{x}(\tau) \in \mathbb{R}^{3}$ that is continuously differentiable, where $\tau \in[0,1]$. The mapping $\mathbf{x}(\tau)$ is one-to-one so that a fiber does not intersect itself. The length measure $l$ of a fiber is

$$
l(\mathrm{~B})=\int_{0}^{1} \mathbf{1}_{\mathrm{B}}(l(\tau))\left|\frac{d \mathbf{x}}{d \tau}\right| d \tau
$$

where $\mathbf{1}_{\mathrm{B}}(l(\tau))$ is the indicator function defined, on a Borel set $\mathrm{B}$, to be unity on $l$ and zero otherwise. A fiber system $L$ is a closed subset of $\mathbb{R}^{3}$, which is the union of countably many fibers $l^{(i)}$ with the property that a compact set is intersected by only a finite number of the fibers. Distinct fibers within a fiber system has only end points in common. This definition thus permits a system of networked dislocations to be modeled as a fiber system. The length measure of a fiber system is defined by

$$
\mathrm{L}(\mathrm{B})=\sum_{l^{(i)} \in \mathrm{L}} l^{(i)}(\mathrm{B})
$$

Obviously, the representation of $\mathrm{L}$ as a union of (locally finite) fibers is not unique but all of the statistically relevant measures are not affected by such non-uniqueness. The family of all fiber systems is denoted by $\mathbb{F}$ and it is endowed with a $\sigma$-algebra $\mathcal{F}$ generated by sets of the form $\{\mathrm{L} \in \mathbb{F}: \mathrm{L}(\mathrm{B})<q\}$ for a Borel set $\mathrm{B}$ and some real number $q$. A fiber process is a random variable $\Phi$ with values in $[\mathbb{F}, \mathcal{F}]$, that 
is, $\Phi$ is a random choice of one of the fiber systems in $\mathbb{F}$. The distribution $\mathrm{P}$ of the fiber process is the probability measure generated on $[\mathbb{F}, \mathcal{F}]$ by $\Phi$. A stationary fiber process is invariant with respect to translation and an isotropic process is invariant with respect to rotation. The intensity measure $\Lambda(B)$ is the expectation value $\mathrm{E}$ of the random length measure $\Phi(\mathrm{B})$, that is

$$
\Lambda(\mathrm{B})=\mathrm{E}(\Phi(\mathrm{B}))=\mathrm{E}\left(\sum_{l^{(i)} \in \mathrm{L}} l^{(i)}(\mathrm{B})\right)
$$

which is interpreted as the mean value of $\mathrm{L}$ over the family of fiber systems $\mathbb{F}$. We note that the family of fiber systems $\mathbb{F}$ plays the role of a statistical ensemble, having the restriction that the length measure $\mathrm{L}$ of the fiber system members of $\mathbb{F}$ is bounded. For a stationary random process, the invariance with respect to translation implies that

$$
\Lambda(\mathrm{B})=I \nu(\mathrm{B})
$$

where $I$ is the intensity of the fiber process and $\nu(\mathrm{B})$ is the Lebesgue measure of B. In the context of crystal dislocations, $I$ is interpreted as the mean dislocation length per unit volume in $\mathbb{R}^{3}$ and $\nu(\mathrm{B})$ defines the volume over which dislocations are distributed.

Consideration of the tangent of the fibers leads to the definition of the rose of directions of the fiber process $\Phi$, which is a measure on the set $\mathbb{T}$ of all directions passing through the origin. Let $\mathbf{t} \in \mathbb{T}$ and $\mathcal{T}$ be the $\sigma$-algebra over $\mathbb{T}$ which are the unions of lines through the origin. For a stationary fiber process, the rose of directions $\mathcal{R}$ is defined by

$$
I v(\mathrm{~B}) \mathcal{R}(\mathrm{A})=\mathrm{E}\left(\int_{\mathrm{B}} \mathbf{1}(\mathbf{t}(\mathbf{x}) \in \mathrm{A})(\mathbf{x}) \Phi(d \mathbf{x})\right) \quad \text { for } \mathrm{A} \in \mathcal{T},
$$

where $\mathbf{t}(\mathbf{x})$ is the tangent to the fiber at $\mathbf{x}$. In Ref. [15], the set $\mathbb{T}$ contains orientations in the upper half sphere of orientations (polar angle $\phi \in[0, \pi / 2]$ and azimuth $\theta \in$ $[0,2 \pi)$ ), thus no distinction is made between an orientation and its opposite. In the case of dislocations, however, the elastic field of the dislocations and processes such as the dislocation climb and cross slip depend on the line direction. Hence, 
two dislocation segments of opposite line directions are not equivalent and, therefore, the entire sphere of orientations $(\phi \in[0, \pi]$ and $\theta \in[0,2 \pi))$ must be considered in computing the rose of directions. Another important point to mention here is that, in the case of infinitesimal deformations, the dislocation lines are planar curves distributed in $3 \mathrm{D}$ space. The intensity is thus interpreted as the average line length per unit volume and the Lebesgue measure is the volume of the crystal space within which dislocations are distributed. The concept of planar fiber process, however, deals with systems of planar curves distributed in a plane, where the intensity is the average length per unit area and the Lebesgue measure is the area of the domain over which the curves are distributed.

\subsection{A simple illustration}

The above definitions are illustrated here by a simple example. Consider a system of dislocation lines $\mathrm{L}$ with members $l^{(i)} \in \mathrm{L}$. Let these lines be contained in a 3D crystal space B that has the form of a cube with side $h$. The Lebesgue measure of B is the volume of the cube $\nu=h^{3}$. Assume that there is only one system of dislocation lines in the family $\mathbb{F}$. Let each of these lines be parameterized by the scalar distance $\ell$ along the dislocation line. The length measure of a single line and of the system of dislocations are

$$
l(\mathrm{~B})=\int_{L} d \ell \quad \text { and } \quad \mathrm{L}(\mathrm{B})=\sum_{l^{(i)} \in \mathrm{L}} \int_{l^{(i)}} d \ell .
$$

The intensity measure $\Lambda(B)$ in this case is equal to $L(B)$ because the expectation value is computed over one system, the given system $L$. The intensity is then given by $I=\Lambda(\mathrm{B}) / \nu=\mathrm{L}(\mathrm{B}) / h^{3}$. The rose of directions is give by

$$
\mathcal{R}(\boldsymbol{l})=\frac{\sum_{l^{(i)} \in \mathrm{L}} \int_{l^{(i)}} \mathbf{1}(\mathbf{t}(\ell) \in \Delta \Omega(\boldsymbol{l})) d \ell}{\Delta \Omega \sum_{l^{(i)} \in \mathrm{L}} \int_{l^{(i)}} d \ell},
$$

where $\Delta \Omega(\boldsymbol{l})$ is a small solid angle element at orientation $\boldsymbol{l}$ having the same value for all orientations, and $\mathbf{t}$ is the line tangent.

Now consider a dislocation loop system $\mathrm{L}=\left\{\right.$ loops of radii $\left.r^{(i)} ; i=1,2, \ldots, n\right\}$ residing on slip planes with normal along the $x_{3}$ direction in a $3 \mathrm{D}$ crystal space 
B that has the form of a cube with side $h$. Let the line orientation for all loops be counter clockwise in the $x_{1} x_{2}$-plane. All unit vectors passing through the origin have the components $(\sin \phi \cos \theta, \sin \phi \sin \theta$ and $\cos \phi)$ along the $x_{1}, x_{2}$ and $x_{3}$-axes, respectively, where $\phi$ and $\theta$ are the usual spherical coordinates, with $\phi$ being the angle between the unit vector and the $x_{3}$-axis. The dislocation line length of a loop $l^{(i)}$ is $2 \pi r^{(i)}$ and $\mathrm{L}=\sum_{i=1}^{n} 2 \pi r^{(i)}$. Consider an orientation in the $x_{1} x_{2}$-plane that is determined by $\phi=\pi / 2$ and some angle $\theta$. A solid angle element $\Delta \Omega=\sin \phi \Delta \phi \Delta \theta$ around that orientation reduces to $\Delta \phi \Delta \theta$ since $\sin \phi=1$. Because the loops lie in the $x_{1} x_{2}$-plane, all segments with orientation within $\Delta \Omega$ are localized at $\phi=\pi / 2$ and their density is proportional to $\Delta \phi$. On each loop thus the line length of interest is $\delta(\phi-\pi / 2) \Delta \phi r^{(i)} \Delta \vartheta$, where $\vartheta=\theta-\pi / 2$ is the polar angle in the plane of the loops measured counterclockwise relative to the $x_{1}$-axis. Implementing equation (10) for the rose of orientation leads to

$$
\mathcal{R}(\phi, \theta)=\frac{\delta(\phi-\pi / 2) \Delta \phi \sum_{i=1}^{n} r^{(i)} \Delta \theta}{\Delta \phi \Delta \theta \sum_{i=1}^{n} 2 \pi r^{(i)}}=\delta(\phi-\pi / 2) \frac{1}{2 \pi} .
$$

This is an example of anisotropic distribution, although it is isotropic with respect to $\theta$. It is easy to show that

$$
\int_{0}^{\pi} d \phi \int_{0}^{2 \pi} d \theta \sin \phi \mathcal{R}(\phi, \theta)=1
$$

If the loops are of irregular shapes but still lie in the $x_{1} x_{2}$-plane, the rose of orientation will have the form $\mathcal{R}(\phi, \theta)=\delta(\phi-\pi / 2) f(\theta) / 2 \pi$.

\subsection{Dislocation correlations}

The expectation value $\mathrm{E}(\Phi(\mathrm{B}))$ of the fiber process defines the measure $\Lambda(\mathrm{B})$ as a

first moment of the random variable $\Phi(\mathrm{B})$. According to [15] higher order moments can also be defined. Of particular interest is the second order moment

$$
\mu^{(2)}=\mathrm{E}\left(\Phi\left(\mathrm{B}_{1}\right) \Phi\left(\mathrm{B}_{2}\right)\right)
$$

which can be used to define the pair correlation functions in fiber processes. Disregarding the line orientation and assuming a stationary and isotropic fiber process, 
a pair correlation function of dislocations $g(\Delta r)$ given only in terms of the scalar separation distance $\Delta r$ has been defined by

$$
\mathrm{E}\left(\mathrm{L}\left(\mathrm{B}_{1}\right) \mathrm{L}\left(\mathrm{B}_{2}\right)\right)=\left\langle\mathrm{L}\left(\mathrm{B}_{1}\right) \mathrm{L}\left(\mathrm{B}_{2}\right)\right\rangle=g(\Delta r)\left\langle\mathrm{L}\left(\mathrm{B}_{1}\right)\right\rangle\left\langle\mathrm{L}\left(\mathrm{B}_{2}\right)\right\rangle=I^{2} \nu\left(\mathrm{B}_{1}\right) \nu\left(\mathrm{B}_{2}\right)
$$

for a pair of small regions $\mathrm{B}_{1}$ and $\mathrm{B}_{2}$ separated by distance $\Delta r$, where $<\cdot>$ refers to the ensemble average of the enclosed quantity. According to Ref. [17] the above definition of the correlation function cannot completely solve the problem of geometric description of the dislocation system.

Consideration of the line orientation in evaluating the dislocation correlations is an important part of the 3D kinetic theory of dislocation [14]. In this theory, evolution of the dislocation system is described by a coupled set of kinetic equations of the form

$$
\left(\partial / \partial t+\mathbf{v} \cdot \nabla+\langle\dot{\mathbf{v}}\rangle \cdot \nabla_{\mathbf{v}}\right)\left\langle\psi^{(n)}\right\rangle=\left\langle S^{(n)}\right\rangle-\left\langle\delta \dot{\mathbf{v}} \cdot \nabla_{\mathbf{v}} \delta \psi^{(n)}\right\rangle ; \quad n=1,2, \ldots, N
$$

where $\psi^{(n)}(\mathbf{x}, \mathbf{v}, \boldsymbol{l}, t)$ is the phase density of dislocations on the $n^{\text {th }}$ slip system, and $\delta \dot{\mathbf{v}}$ and $\delta \psi^{(n)}$ are deviations of the acceleration and phase density from their ensemble averages, respectively. The right hand side of (15) contains the dislocation correlations, both in the first (source) and second (collision) terms. The source terms consist of the cross slip and dislocation reaction terms. Ensemble averaging of the cross slip terms does not give rise to correlations, but averaging of the reaction terms do [14]. Specifically, the treatment of the latter terms give rise to quantities of the form

$$
\left\langle\psi^{(n)} \psi^{(m)}\right\rangle=\left\langle\psi^{(n)}\right\rangle\left\langle\psi^{(m)}\right\rangle\left[1+\zeta^{(n m)}\right] .
$$

where $\zeta^{(n m)}=\zeta^{(n m)}\left((\mathbf{x}, \mathbf{v}, \boldsymbol{l})^{n},(\mathbf{x}, \mathbf{v}, \boldsymbol{l})^{m}, t\right)$ is the correlation function for dislocations on the $n^{t h}$ and $m^{t h}$ slip systems. The importance of these correlations is explained in detail elsewhere [14]. In a general construction of the phase space, the correlations are thus functions over 16D space. By integrating over the velocity part of the phase space, equation (15) reduces to

$$
\frac{\partial\left\langle\varrho^{(n)}\right\rangle}{\partial t}+\nabla \cdot\left(\overline{\mathbf{v}}\left\langle\varrho^{(n)}\right\rangle\right)=\left\langle\mathcal{S}^{(n)}\right\rangle ; \quad n=1,2, \ldots, N
$$

where $\overline{\mathbf{v}}$ is the mean velocity field. The right hand side now contains terms of the form

$$
\left\langle\varrho^{(n)} \varrho^{(m)}\right\rangle=\left\langle\varrho^{(n)}\right\rangle\left\langle\varrho^{(m)}\right\rangle\left[1+\zeta^{(n m)}\right]=\left\langle\varrho^{(n)}\right\rangle\left\langle\varrho^{(m)}\right\rangle g^{(n m)}
$$


in which $\zeta^{(n m)}=\zeta^{(n m)}\left((\mathbf{x}, \boldsymbol{l})^{n},(\mathbf{x}, \boldsymbol{l})^{m}, t\right)$, which are now functions over 10D space. We note here that the unit vector $\boldsymbol{l}$ in 3D is defined by two angles $\phi$ and $\theta$.

In principle, the correlation function defined by equation (18) can be computed using a dislocation dynamics simulation model, for example, the ParaDis code (see section 3.1), but it will be difficult to interpret or visualize the results. For this reason, the correlations are computed in terms of the radial distance $\Delta r=\left|\mathbf{x}^{n}-\mathbf{x}^{m}\right|$ and the two angular differences $\Delta \phi=\left|\phi^{n}-\phi^{m}\right|$ and $\Delta \theta=\left|\theta^{n}-\theta^{m}\right|$ in the current investigation. This amounts to the assumption that the dislocation distribution is statistically homogenous, which is motivated by the use of periodic boundary conditions in the ParaDis model used to conduct the simulations. As shown later, the function $g^{(\alpha \beta)}(\Delta r, \Delta \phi, \Delta \theta)$, rather than $\zeta^{(\alpha \beta)}(\Delta r, \Delta \phi, \Delta \theta)$, will be computed.

It is possible to model the entire dislocation system by a single stochastic fiber process. However, the distinction between the dislocations on various slip systems must be maintained in order to understand the slip system interactions. This does not constitute a difficulty since it is possible to model the entire dislocation system using a set of stochastic fiber processes, one per slip system, with corresponding random variables $\Phi^{(n)} ; n=1,2, \ldots, N$, such that

$$
\mathrm{E}\left(\Phi^{(n)}\right)=\Lambda^{(n)}=\left\langle\varrho^{(n)}\right\rangle \nu
$$

In the above equation $\left\langle\varrho^{(n)}\right\rangle$ plays the role of the intensity of the stochastic fiber process, and $\nu$ is the crystal volume.

\subsection{Dislocation velocity and flux statistics}

In addition to the spatial and orientation statistics discussed above, it is important to understand the velocity and dislocation flux statistics since they are directly related to the plastic strain rate parts carried by dislocation families of various orientations. There is, however, a number of ways to study these statistics. Consider again a system of dislocation lines $\mathrm{L}=\cup_{n=1}^{N} \mathrm{~L}^{(n)}$, where $N$ is the number if slip systems, with members $l^{(i)} \in \mathrm{L}$, each of which being parameterized by the length $\ell$ and contained in a $3 \mathrm{D}$ crystal space B. The simplest possible representation of the velocity statistics 
is given by the set of functions $f^{(n)}(\mathrm{v})=f^{(n)}(|\mathbf{v}|)$ defined by

$$
f^{(n)}(\mathrm{v})=\frac{\sum_{l^{(i)} \in \mathrm{L}^{(n)}} \int_{l^{(i)}} \mathbf{1}(|\mathbf{v}(\ell)| \in \Delta \mathrm{v}(\mathrm{v})) d \ell}{\Delta \mathrm{v} \sum_{l^{(i)} \in \mathrm{L}^{(n)}} \int_{l^{(i)}} d \ell},
$$

where $\Delta \mathrm{v}(\mathrm{v})$ is a finite but arbitrarily small interval about speed $\mathrm{v}$. The velocity spectrum for the entire crystal is defined by

$$
f(\mathrm{v})=\frac{1}{N} \sum_{n=1}^{N} f^{(n)}(\mathrm{v})
$$

The function $f(\mathrm{v})$ has the meaning that $I f(\mathrm{v}) d \mathrm{v}$ is the total dislocation length in the entire crystal having velocity within the range $d \mathrm{v}$ about $\mathrm{v}$, with $I$ being the intensity or the total dislocation length in the crystal.

In order to take into consideration the orientation of the velocity, we define two types of rose of orientations. The first is associated with the velocity, denoted $\mathcal{R}_{\mathbf{v}}^{(n)}(\boldsymbol{l})$, and is defined as follows:

$$
\mathcal{R}_{\mathbf{v}}^{(n)}(\boldsymbol{l})=\frac{\sum_{l^{(i)} \in \mathrm{L}^{(n)}} \int_{l^{(i)}} \mathbf{1}(\boldsymbol{\eta}(\ell) \in \Delta \Omega(\boldsymbol{l})) d \ell}{\Delta \Omega \sum_{l^{(i)} \in \mathrm{L}^{(n)}} \int_{l^{(i)}} d \ell}, \quad \text { for } \quad n=1,2, \ldots, N
$$

in which $\boldsymbol{\eta}=\mathbf{v} / \mathrm{v}$ is a unit vector in the direction of the dislocation velocity. $\mathcal{R}_{\mathbf{v}}^{(n)}(\boldsymbol{l})$ is thus defined such that $I^{(n)} \mathcal{R}_{\mathrm{v}}^{(n)}(\boldsymbol{l}) d \Omega(\boldsymbol{l})$ is the total dislocation length with velocity direction contained in the differential solid angle element $d \Omega(\boldsymbol{l})$ on the $n^{\text {th }}$ slip system. In the special case of conservative motion where dislocations are restricted to glide in their well-defined slip planes, the velocity roses of directions of the individual slip systems can be obtained from the (density) rose of directions by a $\pi / 2$ rotation about their respective slip plane normals.

The second rose of orientations is denoted by $\mathcal{R}_{F}^{(n)}(\boldsymbol{l})$. It is associated with the dislocation flux and defined by

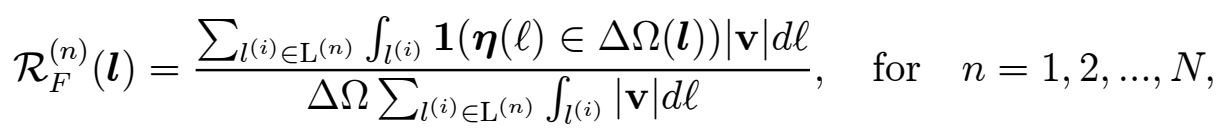

which means that $\dot{\mathcal{A}}^{(n)} \mathcal{R}_{F}^{(n)}(\boldsymbol{l}) d \Omega(\boldsymbol{l})$ is the rate of area swept by dislocations with velocity direction in the differential solid angle $d \Omega(\boldsymbol{l})$ on the $\alpha^{\text {th }}$ slip system, where $\dot{\mathcal{A}}^{(n)}$ is the total rate of area swept by dislocations moving in all orientations on the 
same slip system. It is worth noting here that the velocity and flux roses of directions, given respectively by equations (22) and (23), can be redefined with the orientation $\boldsymbol{l}$ being the dislocation line direction instead of being the direction of the dislocation velocity.

The distributions $f^{(n)}(\mathrm{v}), \mathcal{R}_{\mathbf{v}}^{(n)}(\boldsymbol{l})$ and $\mathcal{R}_{F}^{(n)}(\boldsymbol{l})$ are connected with the shear strain rate on the $n^{\text {th }}$ slip system. For example, the average shear strain rate over a volume $\nu$ on the $n^{\text {th }}$ slip system is given in terms of $f^{(n)}(\mathrm{v})$ by

$$
\overline{\dot{\gamma}}^{(n)}=\left|\mathbf{b}^{(n)}\right|\left\langle\varrho^{(n)}\right\rangle \int_{\mathrm{v}} \mathrm{v} f^{(n)}(\mathrm{v}) d \mathrm{v}
$$

It is also given in terms of $\mathcal{R}_{\mathbf{v}}^{(n)}(\boldsymbol{l})$ and $\mathcal{R}_{F}^{(n)}(\boldsymbol{l})$ by

$$
\begin{aligned}
\dot{\dot{\gamma}}^{(n)} & =\left|\mathbf{b}^{(n)}\right|\left\langle\varrho^{(n)}\right\rangle \int_{\Omega}|\mathbf{v}(\boldsymbol{l})| \mathcal{R}_{\mathbf{v}}^{(n)}(\boldsymbol{l}) d \Omega(\boldsymbol{l}) \\
& =\left|\mathbf{b}^{(n)}\right|\left\langle\varrho^{(n)}\right\rangle \int_{\Omega} \overline{\mathbf{v}}_{\Omega} \mathcal{R}_{F}^{(n)}(\boldsymbol{l}) d \Omega(\boldsymbol{l}),
\end{aligned}
$$

where $\mathbf{b}^{(n)}$ is the Burgers vector of the $n^{\text {th }}$ slip system and $\overline{\mathrm{v}}_{\Omega}$ is the average over all orientations of the mean dislocation speed on the same slip system. The choice of one expression of the shear strain rate over the others is then dictated by the kind of information available.

\section{Numerical simulation}

\subsection{The dislocation dynamics model}

The ParaDis dislocation dynamics model, see [16, 18] for details, has been used to obtain numerical data to implement the definitions developed in section 2 . In the ParaDis model, the dislocation configuration is a collection of piece-wise linear segments connected at nodes. This configuration is evolved by integrating the nodal velocities with respect to time. The velocities are computed using a force-velocity law in which the (Peach-Koehler) force is computed either as the negative gradient of the energy of the dislocation system with respect to the nodal coordinates, or by the integral over segments incident with each node, of the point-wise forces on the 
segments times some weight function. Non-singular expressions of the dislocation stress field and energy that have been recently derived are used for this purpose [18]. In the ParaDis code version used in conjunction with this study, dislocations of screw character do not have well defined glide planes in body-centered cubic crystals. As a consequence, the dislocation segments connected to those of screw character can in some cases move non conservatively. In the latest version of the ParaDis code, however, dislocations execute conservative motion only [19]. In all ParaDis versions, periodic boundary conditions are employed.

\subsection{Simulation methodology}

A body-centered cubic crystal is considered in the present study, which has slip systems of the type $a / 2<111>\{110\}$, with the magnitude of the Burgers vector $b=2.725 \AA$. This family of Burgers vectors include $a / 2[111], a / 2[11 \overline{1}], a / 2[1 \overline{1} 1]$, and $a / 2[1 \overline{1} \overline{1}]$, which are denoted here by $\mathrm{b} 1, \mathrm{~b} 2, \mathrm{~b} 3$ and $\mathrm{b} 4$, respectively. The crystal is loaded in the $[100]$ direction at constant strain rate. Two values of the strain rate $\dot{\varepsilon}$ have been used, $\dot{\varepsilon}_{1}=10 \mathrm{~s}^{-1}$ and $\dot{\varepsilon}_{2}=1 \mathrm{~s}^{-1}$, corresponding to the higher and lower stress-strain curves in Figure 2, respectively. Numerical data has been collected at four strain levels on each of the two curves in order to study the effect of both the strain and its rate on the evolution of the statistical quantities of the dislocation system. On the high stress-strain curve, data has been collected at strain values of 0.0025, 0.005, 0.0096 and 0.013. These points are denoted by H1, H2, H3 and H4, respectively. On the lower stress-strain curve, data has been collected at strain values of $0.0012,0.0075,0.015$ and 0.022 , which are denoted by L1, L2, L3 and L4, respectively. The crystal simulation volume is of the form of a cube with edge length of $17.5 \times 10^{3} \mathrm{~b}$.

\section{Add figure 2 here.}

The numerical data collected includes the nodal coordinates, segment connectivity, line sense, and the nodal and segment velocities, which was then used to analyze the orientation, spatial, velocity and flux statistics in accordance with the expressions presented in the previous section. In constructing $\mathcal{R}(\boldsymbol{l}), \mathcal{R}_{\mathbf{v}}(\boldsymbol{l}), \mathcal{R}_{F}(\boldsymbol{l})$ and 


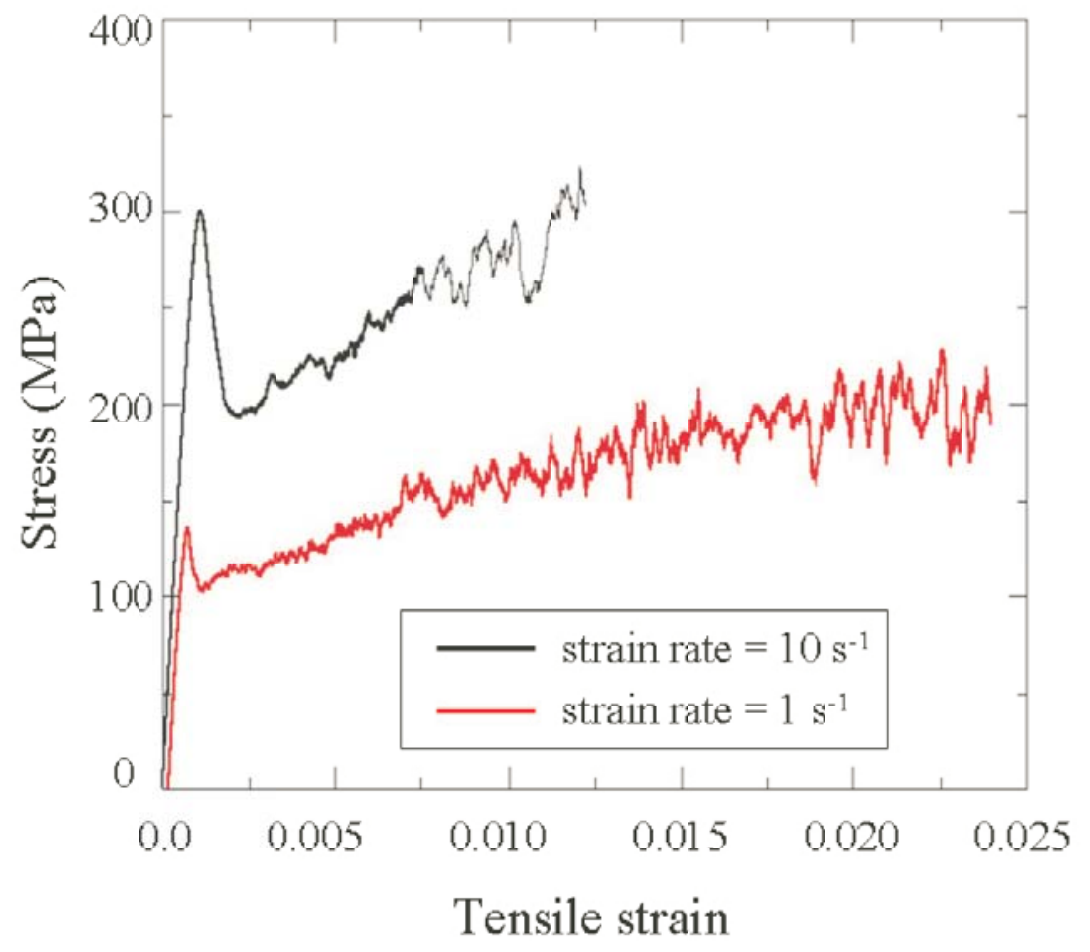

Figure 2: Stress-strain curve of a Nb crystal loaded along the [100] direction at two strain rates, $10 \mathrm{~s}^{-1}$ (higher curve) and $1 \mathrm{~s}^{-1}$ (lower curve). All Burgers vectors are active. 
$g(\Delta r, \Delta \phi, \Delta \theta)$, the angular coordinates have been discretized into 18 and 36 intervals for $\phi$ and $\theta$, respectively. The maximum spatial range considered in computing the dislocation correlations is $17.5 \times 10^{3} \mathrm{~b}$, which is the same as the size of the simulation box. In implementing the formula (18) for the correlation function $g$, the box has been divided into $10 \times 10 \times 10$ elements and replicated is all directions to avoid any edge effects.

\subsection{Results and discussion}

Figure 3 shows the evolution of the rose of directions of dislocations with Burgers vector b1 at the points $\mathrm{H} 1, \mathrm{H} 2$ and $\mathrm{H} 3$ on the high strain rate curve in Figure 2. The initial rose of directions shows that the density is localized on the three cross slip planes sharing the Burgers vector b1. As the strain increases, and due to the nonconservative motion considered in the ParaDis model used in this study, the rose of directions spreads to fill orientations that do not belong to the three cross slip planes associated with b1. Moreover, the density grows preferentially along the direction of the Burgers vector as the strain increases, indicating a buildup of screw dislocation line density. All the three cross slip planes contribute to the screw orientation peaks because this orientation is shared by these planes.

A comparison between the rose of directions of dislocations of Burgers vectors b1, b2 and b3 at point $\mathrm{H} 4$ is shown in Figure 4. Again, in these roses, peaks follow the directions of the Burgers vectors, indicating buildup of the screw density in all cases. During the course of this study, it has been also found that the evolution of the rose of directions exhibits strong dependence on the strain rate. In particular, at lower strain rate the screw orientation peaks have been found to be less sharper and the effect of the nonconservative motion is more pronounced. It is anticipated that the evolution of the rose of directions will also depend on the loading direction of the crystal, but this has not been a part of this investigation.

Add figure 3 here.

Add figure 4 here. 

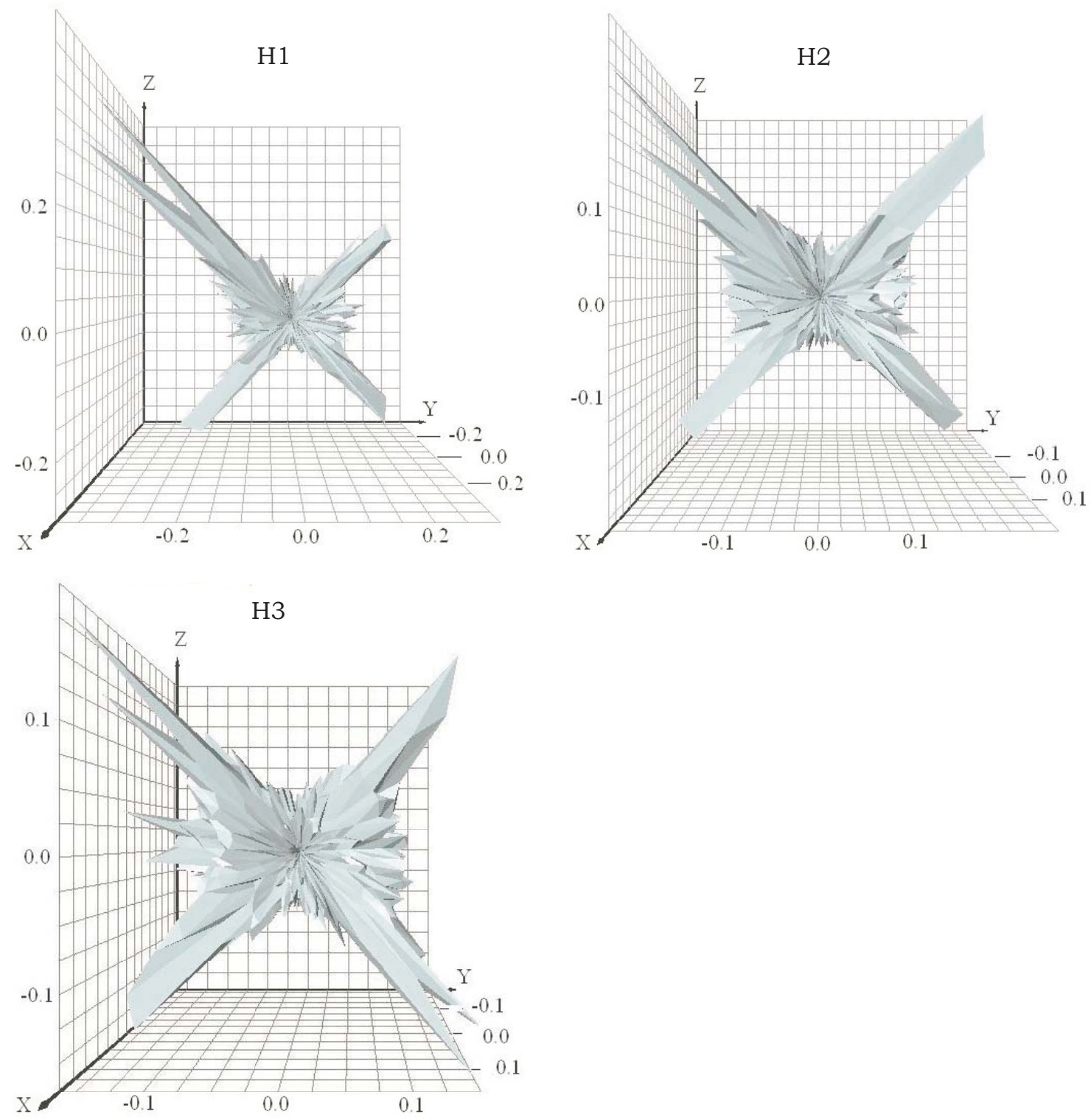

Figure 3: The rose of directions of dislocations with Burgers vector b1 at the points H1, $\mathrm{H} 2$ and $\mathrm{H} 3$. 

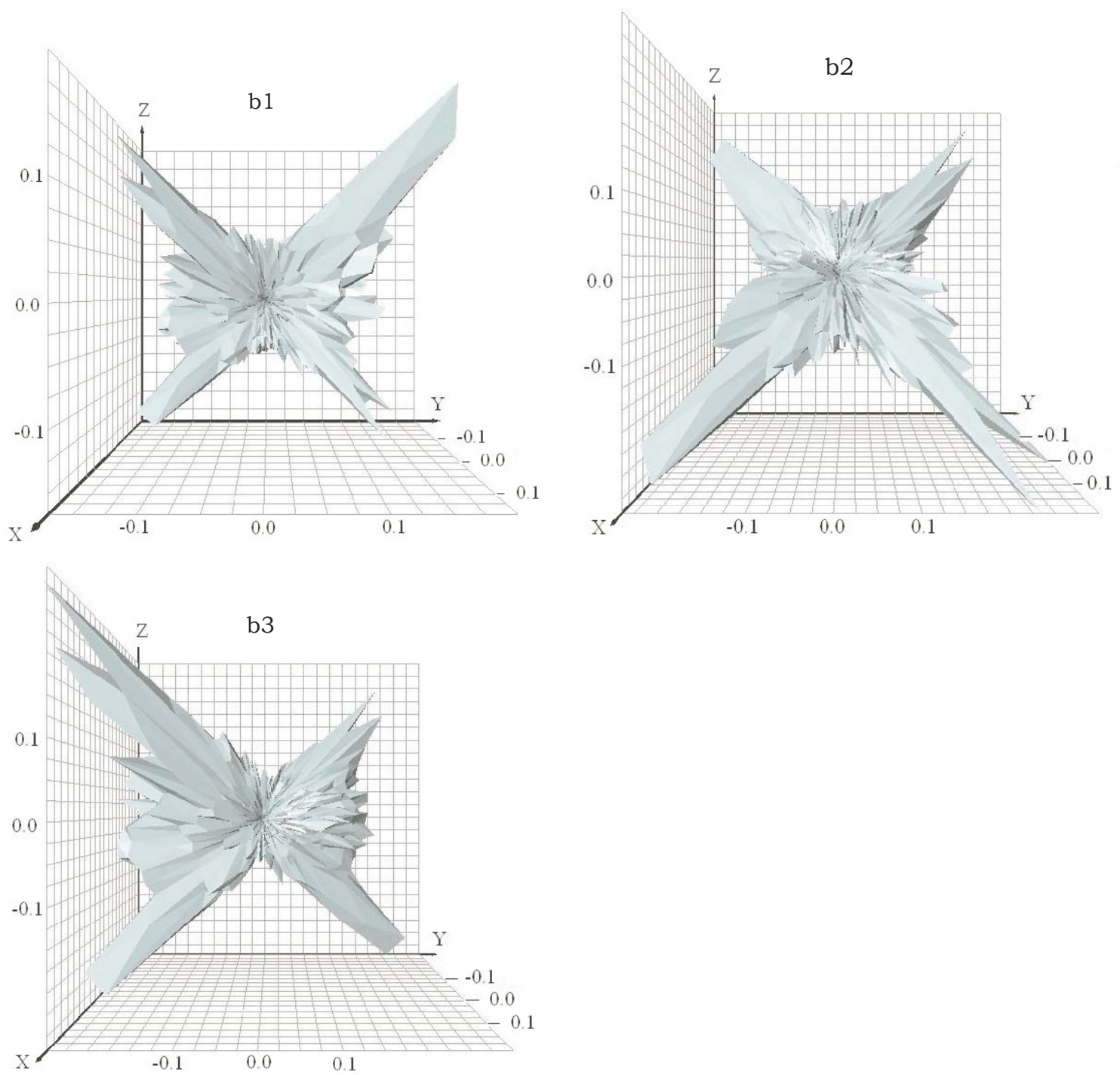

Figure 4: The rose of directions of dislocations with Burgers vector b1, b2 and b3 at the point H4. 
The velocity spectrum has been computed using equations (21) at four points on each of the stress-strain curves shown in Figure 2. Plots of the spectra at these points are shown in Figure 5. In all cases, the velocity spectrum shows a peak close to zero velocity, indicating that a fraction of the dislocation density is sessile or has very low velocity, which is characteristic of dislocation motion in body-centered cubic crystals. These sessile or slowly moving dislocations are either participating in junctions or the force acting on them is below the Peierls barrier. The spectrum also falls off quickly at higher velocity values but maintains a long tail, indicating that a small number of segments aquire velocity far higher than the average. It is also shown that, at higher strain rate, a larger fraction of the dislocations has higher velocity; that is, the average velocity of dislocations increases at higher strain rate, which is consistent with the increased value of the external stress.

Add figure 5 here.

The rose of directions of the dislocation velocity and flux have been computed using equations (22) and (23) for the dislocations sharing the same Burgers vector. Figure 6 shows these rose of directions at the points L2 and L3 on the lower stressstrain curve in Figure 2 for Burgers vector b1. It is noted that these roses of directions peak along the direction of the Burgers vector, which is the direction of the motion of the edge components on all cross slip planes sharing b1 as their Burgers vector. The analysis further shows that these rose of directions exhibit strong dependence on the strain level and strain rate. Because the resolved shear stress on all slip planes depend on the loading configuration of the crystal, it is anticipated that the velocity and flux rose of directions will also strongly depend on the way the crystal is being loaded.

\section{Add figure 6 here.}

Equation (18) for the correlation function has been implemented for dislocations belonging to specific Burgers vector. This selection has been motivated in part by the fact that, in the ParaDis model used here, not all dislocations have well defined 

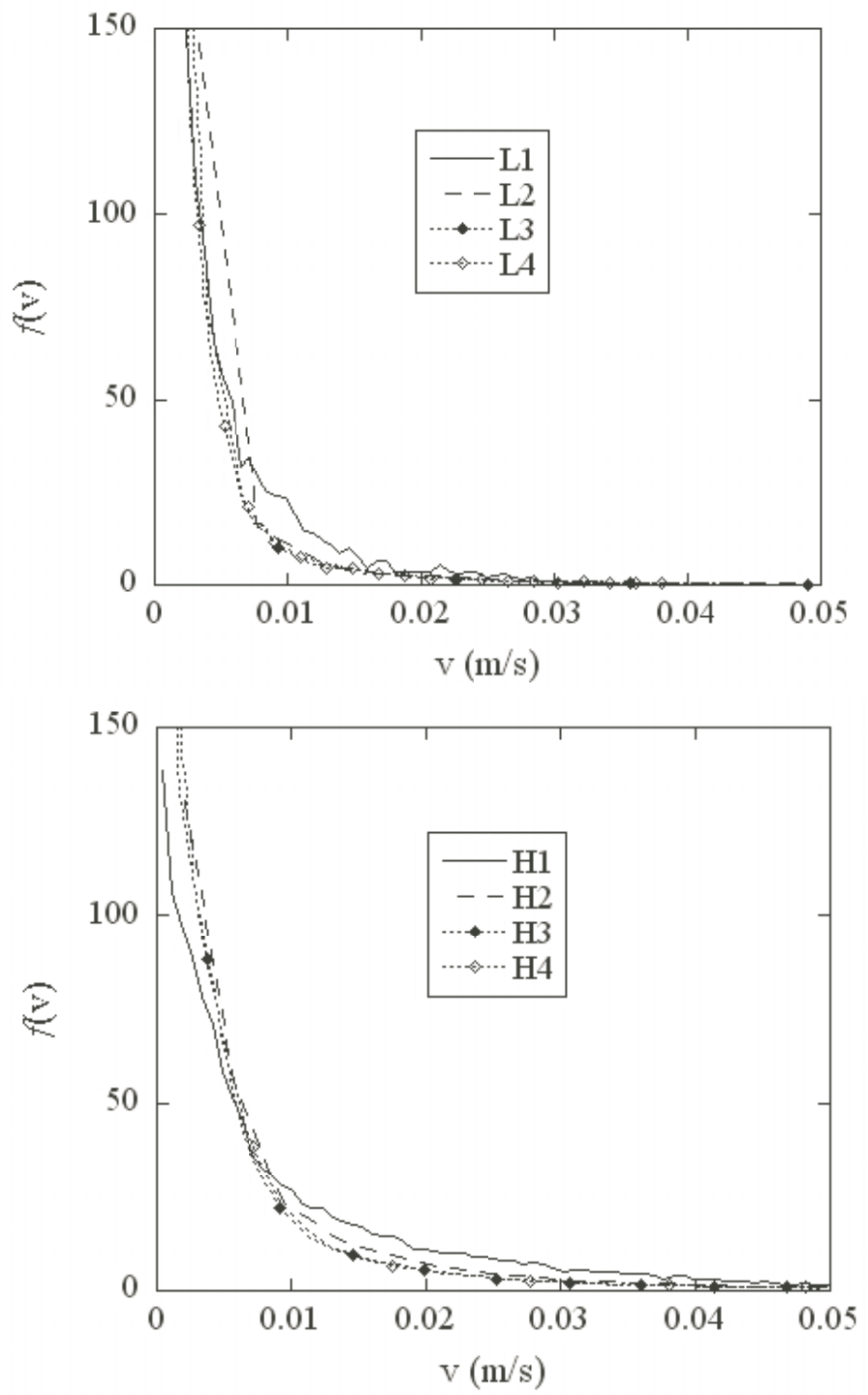

Figure 5: The velocity spectra at the points L1, L2, L3 and L4, and H1, H2, H3 and H4 on the stress-strain curves shown in Figure 2. 

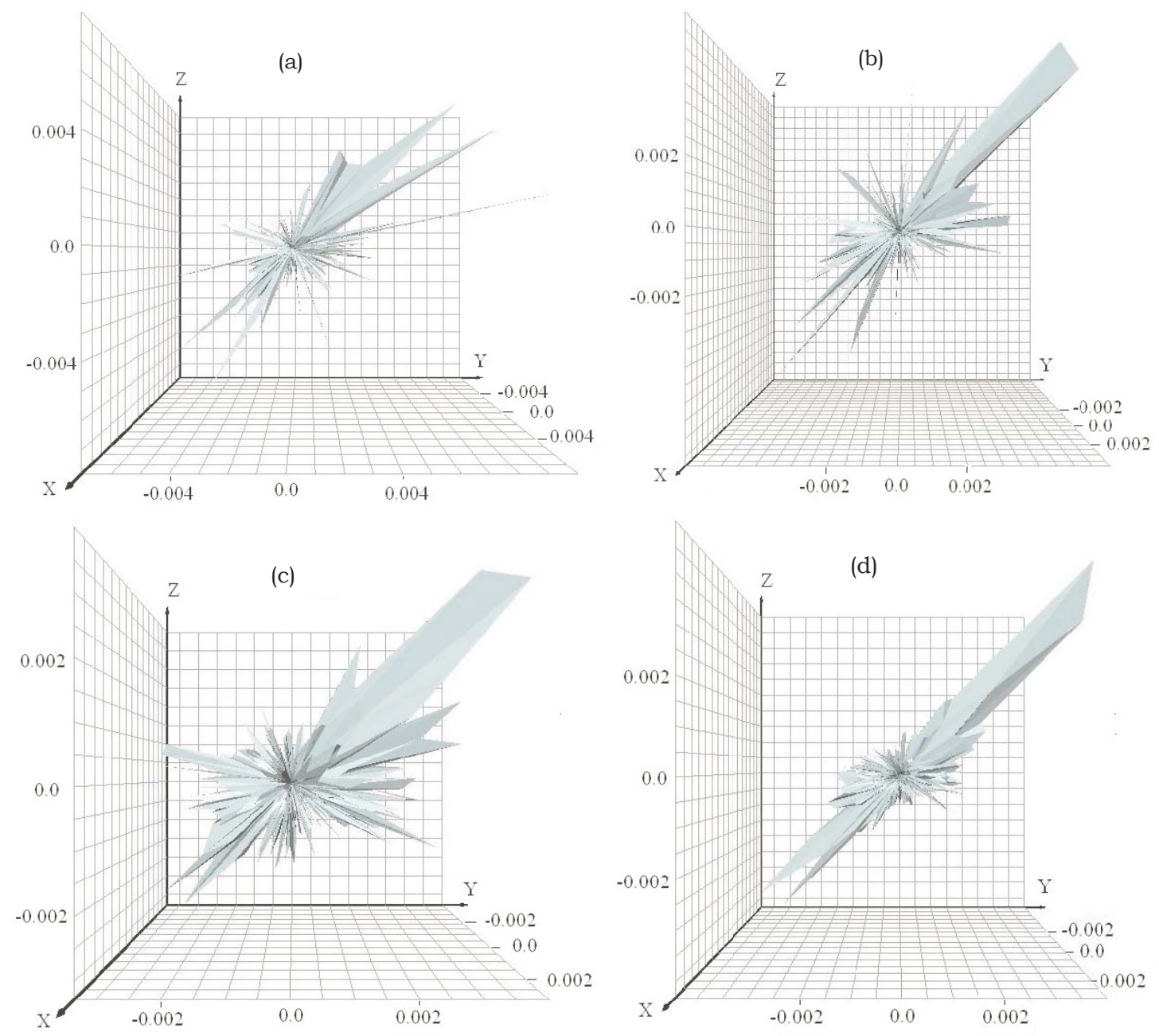

Figure 6: The rose of directions for the dislocation flux (a and b) and velocity (c and d) at points L2 (a and c) and L3 (b and d) of the lower stress-strain curve in Figure 2. 
slip planes and, therefore, dislocations can only be distinguished by their Burgers vector. It has also been motivated by the fact that the formalism treats all segment orientations by two angles $(\theta, \phi)$, irrespective of their Burgers vector or slip planes. Thus, in this study, results are presented for correlations of the form

$$
g^{(\mathrm{bjbj})}=\frac{\left\langle\varrho^{(b j)} \varrho^{(b j)}\right\rangle}{\left\langle\varrho^{(b j)}\right\rangle\left\langle\varrho^{(b j)}\right\rangle},
$$

where $b j$ is an element of the set $\{b 1, b 2, b 3, b 4\}$. The cross correlations between slip systems are left for a future study.

In implementing the expression (26), the ensemble averages of the densities and density pair products have been computed in terms of the appropriate sums of densities and density pair products. Figure 7 shows two sample results of dislocation correlations for b3 and b4 at the point H4 on the higher stress-strain curve in Figure 2. The ranges of $\Delta r, \Delta \theta$ and $\Delta \phi$ in these figures are $17.5 \times 10^{3} \mathrm{~b}, 2 \pi$ and $\pi$, respectively. Figure $7 \mathrm{a}$ shows an isosurface $g^{(\mathrm{b} 3 \mathrm{~b} 3)}=0.316$, an orthoslice at $\Delta r=3.5 \times 10^{3} \mathrm{~b}$ and two other orthoslices at $\Delta \theta=\Delta \phi=\pi / 9$. Figure $7 \mathrm{~b}$ shows an isosurface at $g^{(\mathrm{b} 4 \mathrm{~b} 4)}=0.83$ an orthoslice at $\Delta r=3.5 \times 10^{3} \mathrm{~b}$ and two other slices at $\Delta \theta=\Delta \phi=\pi / 9$. The color code is such that the yellow is the highest numerical value and the gray is the lowest one. While it is difficult to visualize and interpret the simulated dislocation correlations in a space of dimension 3 or higher, a number of preliminary observations have been made during the course of the present study. First, the correlation shows a sharp peak at $(\Delta r, \Delta \theta, \Delta \phi)=(0,0,0)$. This behavior is displayed by the yellow range of color on the orthoslices. Second, the correlation initially decays away from the origin along the three axes. However, this decay continues monotonically only along $\Delta r$ and the correlation rather peaks again in other regions of the $(\Delta \theta \Delta \phi)$-plane, particularly at points that correspond to opposite line sense, which reflects the formation of dislocation dipoles. This observation is consistent with the peaking of the rose of directions at certain orientations and their opposite. Third, the monotonic decay along $\Delta r$-axis resembles a $1 / \Delta r$ behavior over a large portion of the $\Delta r$ range, indicating a possible effect of the long range stress field.

Add figure 7 here. 

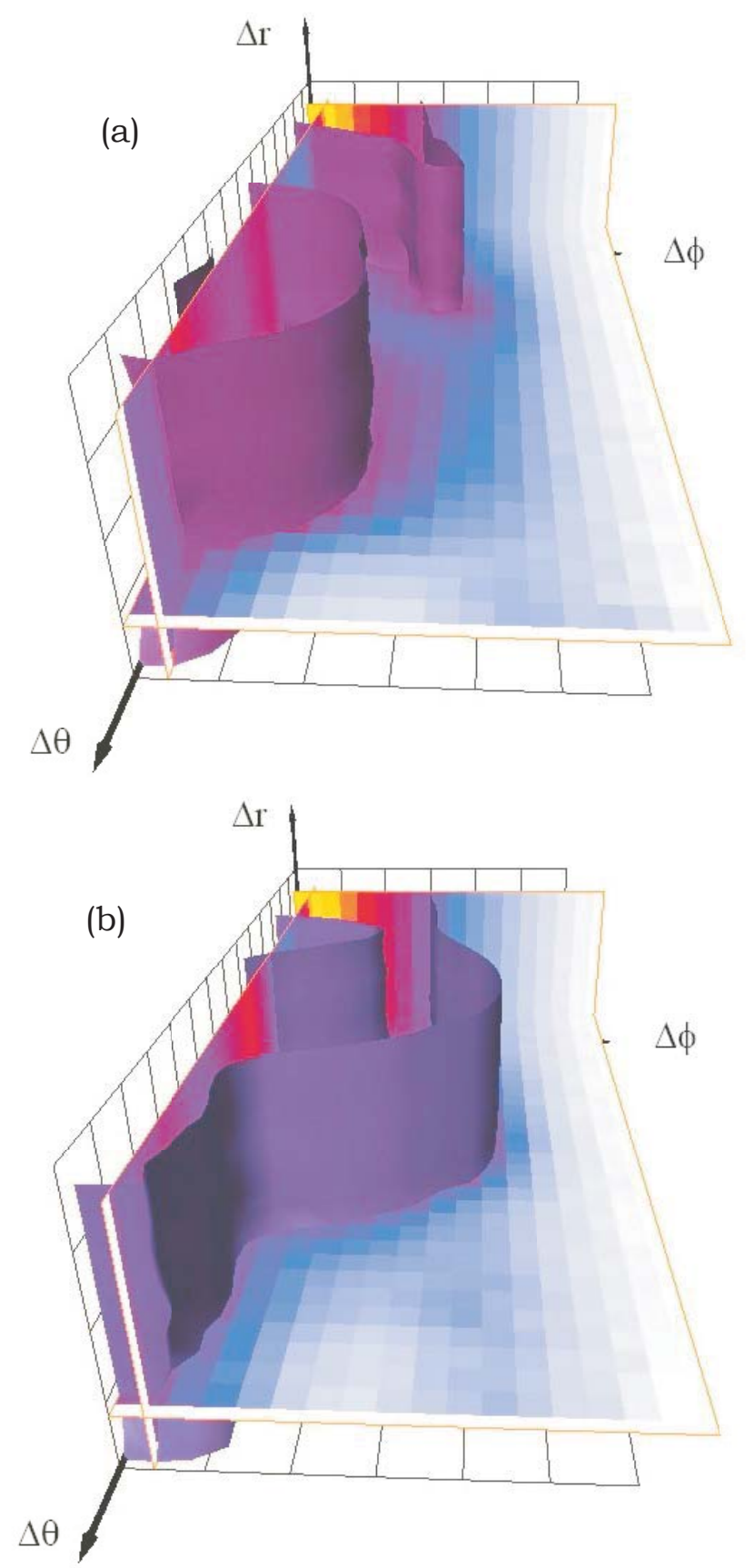

Figure 7: Dislocation correlations for dislocations of Burgers vectors b3 (a) and b4 (b). 


\section{Concluding remarks and future directions}

The present work represents a first step towards a formal treatment of the statistics of dynamical dislocation systems, which is an important part of the statistical framework of modeling the collective behavior of dislocation and mesoscale crystal deformation. A number of statistical dislocation measures have been introduced in the present study, which include three roses of directions that characterize the orientation dependence of the dislocation density, velocity and flux. The dislocation correlation is also introduced as a fourth statistical measure that depends on the spatial and angular distances between pairs of dislocation densities. The connection between these statistical measures and the kinetic evolution of dislocations has been outlined.

The rose of directions used in the present work is the first velocity moment of the phase density measure introduced in the earlier work by El-Azab [11, 14]. This statistical measure has been defined in connection with the theory of stochastic fiber process [15]. An additional part of this theory, which studies the intersection of two stochastic fiber processes, can be further used in modeling dislocation systems. The intersection of two stochastic fiber processes $\Phi^{(n)}$ and $\Phi^{(m)}$ with intensities $I^{(n)}$ and $I^{(m)}$ and rose of directions $\mathcal{R}^{(n)}$ and $\mathcal{R}^{(n)}$, respectively, yields a stochastic point process of intensity $\lambda$ given by

$$
\lambda=I^{(n)} I^{(m)} \mathcal{F}\left[\mathcal{R}^{(n)}(\boldsymbol{l}), \mathcal{R}^{(m)}(\boldsymbol{m}) ; \boldsymbol{l}, \boldsymbol{m}\right],
$$

where $\mathcal{F}[\cdot]$ is a functional of the roses of directions and the unit vectors $\boldsymbol{l}$ and $\boldsymbol{m}$. The intensity $\lambda$ then defines the number of intersection points per unit volume. Assuming that the stochastic fiber processes $\Phi^{(n)}$ and $\Phi^{(m)}$ represent dislocations on two different slip systems, then the intensity of the associated stochastic point process defines points at which special events such as junctions formation, annihilation, or Burgers vector reactions take place. The types of intersection can be distinguished through the definition of the functional $\mathcal{F}$. Furthermore, the spatial distributions of the points of intersection can be analyzed statistically to provide quantitative measures that help model the density of short range reaction events. The fact that the intensity of the 
stochastic point process resulting from the intersection of two fiber processes must be related to the dislocation correlations is intuitive. Further analysis in this direction will thus enable the modeling of the source terms in the kinetic theory presented in $[11,14]$; see also equations (15) and (17). Future elaboration of the theory of stochastic fiber processes in the context of dislocation dynamics and mesoscale plasticity thus seems a promising direction to follow in developing continuum theories.

\section{Acknowledgements}

JD has been supported in part by the LDRD Program of Oak Ridge National Laboratory under the UT - Battelle LLC contract number 4000040123 at Florida State University. The authors thank Tom Arsenlis and Vasily Bulatov of Lawrence Livermore National Laboratory for making the ParaDis code available for use in this study.

\section{Appendix: Borel sets and $\sigma$-algebra}

Stoyan and co-authors [15] make an extensive use of the set theory language to define the stochastic fiber process. In adopting the pertinent definitions after these authors, the terms Borel set and $\sigma$-algebra have been used in this paper. These terms are defined in this appendix.

A $\sigma$-algebra or a $\sigma$-field over a set $\mathrm{S}$ is a collection $\Sigma$ of subsets of $\mathrm{S}$ that is closed under countable set operations. $\sigma$-algebras have developed in order to define measures on a set S; a measure is a function that assigns a number, for example a size, to subsets of a given set, which facilitates integration over the set. $\Sigma$ is a $\sigma$-algebra if and only if it has the following properties: (P1) the empty set is in $\Sigma$, (P2) if $\mathrm{E}$ is in $\Sigma$ then so is its complement $\mathrm{S} \backslash \mathrm{E}$, and (P3) the union of countably many sets in $\Sigma$ is also in $\Sigma$. From the properties $\mathrm{P} 1$ and $\mathrm{P} 2$, it follows that $\mathrm{S}$ is in $\Sigma$, and from P2 and P3 it follows that the $\sigma$-algebra is also closed under countable intersections. Elements of the $\sigma$-algebra are called measurable sets. An ordered pair $[S, \Sigma]$, where 
$\mathrm{S}$ is a set and $\Sigma$ is a $\sigma$-algebra over $\mathrm{S}$, is called a measurable space. A measure $\mu$ on a measurable space $[S, \Sigma]$ define a measure space $[S, \Sigma, \mu]$.

The Borel algebra (or Borel $\sigma$-algebra) on a topological space $\mathrm{S}$ is a $\sigma$-algebra of subsets of $\mathbf{S}$ associated to the topology of $\mathrm{S}$. The elements of the Borel algebra are called Borel sets, which can be constructed from open or closed sets by countable unions and intersections. The class $\mathcal{B}^{d}$ of Borel sets in $\mathbb{R}^{d}$ is the smallest collection of sets that includes open and closed sets such that if $E, E_{1}, E_{1}, \ldots$ are in $\mathcal{B}^{d}$, then so are $\cup_{i=1}^{\infty} \mathrm{E}_{i}, \cap_{i=1}^{\infty} \mathrm{E}_{i}$, and $\mathbb{R}^{d} \backslash \mathrm{E}[20,21]$. $\mathbb{R}^{d}$ the Euclidean space of dimension $d$. For the sake of the present problem, Borel sets indicate crystal regions over which dislocations are distributed. 


\section{References}

[1] Valdo A. Lubarda, Elastoplasticity Theory, (CRC Press, New York, 2002).

[2] K. S. Havner, Finite Plastic Deformation of Crystalline Solids (Cambridge University Press, New York, 1992).

[3] E. Kröner, in Physics of Defects, edited by R. Balian, M. Kléman, and J.-P. Poirier (North-Holland Publishing Company, Amsterdam, 1994), p. 217.

[4] E. Kröner, International Journal of Solids and Structures 291849 (1992).

[5] A. M. Kesevich, Soviet Physics Uspekhi 7837 (1965).

[6] L.P. Kubin, G. Canova G, M. Condat, B. Devincre, V. Pontikis and Y. Brechet, Diffusion and Defect Data-Solid State Data, Part B (Solid State Phenomena) 23-24 455 (1992).

[7] M. Rhee, H. M. Zbib, J. Hirth, H. Huang and T. de al Rubia, Modelling and Simulation in Materials Science and Engineering 6467 (1998).

[8] N.M. Ghoniem and J. Huang, Journal de Physique IV 11 Pr5-53 (2001).

[9] E. Kröner, in Inelastic Behavior of Solids, edited by M. F. Kanninen, W. F. Adler, A. R. Rosenfield and R. I. Jaffee (McGraw-Hill Book Company, New York, 1970), p. 137.

[10] E. Kröner, International Journal of Solids and Structures 381115 (2001).

[11] A. El-Azab, Physical Review B 6111956 (2000).

[12] M. Zaiser, M.-C. Miguel and I. Groma, Physical Review B 64224102 (2002).

[13] I. Groma, Physical Review B 565807 (1997).

[14] A. El-Azab, Scripta Materialia 54723 (2006).

[15] D. Stoyan, W. S. Kendall and J. Mecke, Stochastic Geometry and its Applications, (John Wiley \& Sons, New York, 1995). 
[16] W. Cai and M. Hiratani, ParaDis User Manual, Lawrence Livermore National Laboratory (UCRL-CODE-155933), 2004.

[17] H. Stoyan and D. Stoyan, Physica Status Solidi (a) 97163 (1986).

[18] Wei Cai, Athanasios Arsenlis, Christopher R. Weinberger and Vasily V. Bulatov, Journal of the Mechanics and Physics of Solids 54561 (2006).

[19] Vasily V. Bulatov, Private Communication (2006).

[20] H.T. Croft, K. J. Falconer, and R. K. Guy, Unsolved Problems in Geometry, (Springer-Verlag, New York, 1991).

[21] H. Grimmett and D. Strizaker, Probability and Random Processes, (Oxford University Press, Oxford, 2001). 


\section{Figures captions}

Figure 1. Snapshot of the dislocation distribution in Nb crystal loaded in the [100] direction at constant strain rate of $1 \mathrm{~s}^{-1}($ strain $=0.018)$.

Figure 2. Stress-strain curve of a Nb crystal loaded along the [100] direction at two strain rates, $10 \mathrm{~s}^{-1}$ (higher curve) and $1 \mathrm{~s}^{-1}$ (lower curve). All Burgers vectors are active.

Figure 3. The rose of directions of dislocations with Burgers vector b1 at the points $\mathrm{H} 1, \mathrm{H} 2$ and $\mathrm{H} 3$.

Figure 4. The rose of directions of dislocations with Burgers vector b1, b2 and b3 at the point $\mathrm{H} 4$.

Figure 5. The velocity spectra at the points L1, L2, L3 and L4, and H1, H2, H3 and H4 on the stress-strain curves shown in Figure 2.

Figure 6. The rose of directions for the dislocation flux (a and b) and velocity (c and d) at points L2 (a and c) and L3 (b and d) of the lower stress-strain curve in Figure 2 .

Figure 7. Dislocation correlations for dislocations of Burgers vectors b3 (a) and b4 (b).

This work was performed under the auspices of the U. S. Department of Energy by University of California, Lawrence Livermore National Laboratory under Contract W-7405-Eng-48. 